\title{
The many facets of research integrity: What can we do to ensure it?
}

\author{
Desmond M. D’Souza, MD, ${ }^{a}$ Robert M. Sade, MD, ${ }^{b}$ and Susan D. Moffatt-Bruce, MD, PhD ${ }^{a}$
}

Research misconduct and detrimental research practices damage the foundations of biomedical research. The reliability of research findings and the medical literature that reports them are fundamentally important for ensuring both the provision of good clinical care and the appropriate use of science and technology resources. As scientists and care providers, we assume that we can trust the literature we read and that it reports reliable research findings and conclusions. Protecting the research enterprise is an active process and the responsibly of many individuals and institutions, including the surgeons who might alter their practices based on journal reports, funding bodies, institutions that facilitate and support the research, and ultimately the editorial staff of the journals that disseminate research findings.

\section{WHAT DOES IT MEAN TO PROTECT RESEARCH INTEGRITY?}

Biomedical research is complex, and violations of standards and norms can occur in a wide variety of ways that range from transgressions of personal honesty, such as plagiarism and duplicate publication, to horrifically damaging offenses such as falsification and fabrication of data that undermine the very foundations of science. For example, public health is still reeling from a false report of a nonexistent linkage between the measlesmumps-rubella vaccine and autism in children 2 decades ago. ${ }^{1,2}$ Particularly damaging to individual patients are transgressions, including unethical consenting practices and unsafe treatment of study participants. The spectrum of research misconduct is broad, with substantial implications on many levels. The many facets of scientific integrity and the many parties responsible for protecting it require the use of a variety of interventions to detect and adjudicate misconduct. As scientists and surgeons, we must support effective interventions to ensure the soundness

From the a Division of Thoracic Surgery, Department of Surgery, The Ohio State University, Columbus, Ohio; and ${ }^{\mathrm{b} D i v i s i o n}$ of Cardiothoracic Surgery, Department of Surgery, Institute of Human Values in Health Care, Medical University of South Carolina, Charleston, SC.

Received for publication Nov 29, 2019; revisions received Dec 23, 2019; accepted for publication Dec 30, 2019; available ahead of print March 30, 2020.

Address for reprints: Susan D. Moffatt-Bruce, MD, PhD, Division of Thoracic Surgery, Department of Surgery, The Ohio State University, 168 Doan Hall, 410 W 10th Ave, Columbus, OH 43210 (E-mail: smoffattbruce@ royalcollege.ca).

J Thorac Cardiovasc Surg 2020;160:730-3

$0022-5223 / \$ 36.00$

Copyright $₫ 2020$ Published by Elsevier Inc. on behalf of The American Association for Thoracic Surgery

https://doi.org/10.1016/j.jtcvs.2019.12.127

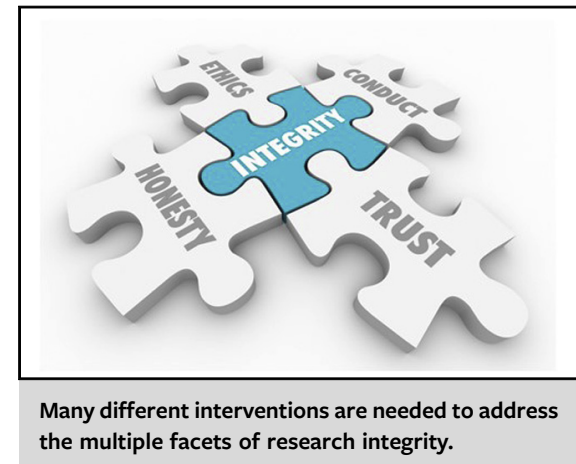

\section{CENTRAL MESSAGE \\ Surgeons and institutions have a central role in protecting and ensuring the integrity of research. Many factors lead to research misconduct, so many different interventions are needed to address them.}

This Invited Expert Opinion provides a perspec tive on the following presentation: https://nexus od.nih.gov/all/2019/08/15/thoughts-on-how-institutions can-promote-a-culture-of-research-integrity/.

See Commentaries on pages 734 and 735 .

of the research that we and our colleagues carry out and that our journals report, thereby ensuring the safety of our patients.

\section{WHAT IS THE MAGNITUDE OF THE PROBLEM?}

Recently, institutions have been urged to take more responsibility for ensuring research integrity. ${ }^{3,4} \mathrm{~A}$ wide range of problems in institutional investigations of allegation of research misconduct have been identified, including poor quality of investigative processes, limited oversight, and variability in the ultimate misconduct reports. Institutions that employ the researchers, own and maintain the facilities in which the research is executed, collect and administer grant funds, and train the next generation of researchers have primary responsibility for ensuring research integrity, yet this institutional obligation has been poorly 
performed. ${ }^{3,4}$ Moreover, research standards and investigative practices and policies are administered by various regulatory agencies, and these standards, practices, and policies vary among countries. ${ }^{5}$ Furthermore, the practices and policies that do exist are rarely evidence-based, and no formal entity either nationally or internationally is ultimately responsible for oversight of research integrity standards. ${ }^{5}$ Clearly, the problem of ensuring scientific integrity is real and pervasive.

The biomedical and life sciences literature has endured thousands of retractions in recent years, mostly for suspected fraud or duplicate publication. ${ }^{6}$ We suspect that the large number of retractions are but the tip of the iceberg, because the reported cases represent only those that are detected and adjudicated; therefore, the overall incidence of research misconduct is likely to be greatly underreported.

The magnitude of the problem was illustrated by the recent report of payment of millions of dollars in fines to federal agencies by multiple institutions for fabricating data. $^{7}$ Whilst the principle investigator is ultimately responsible, maintaining research integrity must also extend to the entire research team that is complicit, often unwittingly, in the deceit. ${ }^{7}$ Investigations of misconduct in more than 200 publications by 4 major academic institutions took 8 to 17 months to complete. ${ }^{8}$ During this prolonged investigative time, flawed data or clinical recommendations could be disseminated and adopted before action could be taken to warn physicians of the defective information.

Three reports from the National Academies of Science, Engineering and Medicine (NASEM) underscore the phenomenon not only of research misconduct, but also of failure to recognize it. ${ }^{9-11}$ During 2016, NASEM reported that "academic research institutions have failed to respond appropriately to investigators' transgressions or failed to use effectively the range of tools available to create an environment that strongly discourages, at both the institutional and the individual level." ${ }^{\text {"9 }}$ During 2017, the NASEM Committee on Responsible Science noted, "Significant gaps exist in the information available to institutions as well as to the rest of the research enterprise about how allegations are handled, what challenges arise and how successful institutions are able to ensure effective performance." ${ }^{10}$ Lastly, the Committee on the Review of Omics-Based Tests for Predicting Patient Outcomes in Clinical Trials, reported that "institutions could be influenced by secondary interests beyond simply the financial ones and could be grouped together under reputational value for the institution and the individual researcher." problem in the detection and investigation of research transgressions, and ultimately in the assumption of responsibility for scientific integrity.

\section{WHY IS THE LOSS OF RESEARCH INTEGRITY SUCH A PROBLEM?}

As a profession, we are responsible for our own code of conduct and for ensuring adherence to it. Fraudulent and unethical research practices and insufficient investigations undermine public confidence in us as scientists and surgeons and negatively influence patient care and affect the development of future technologies. ${ }^{12}$ Research misconduct has grown in the biomedical and broader scientific research arenas during recent decades. Many causes can be ascribed to research misconduct and the resultant loss of integrity, including efforts toward personal promotion, competition for research grants and funding, and competitive forces at the institutional level, such as a race to innovate and disseminate new technologies, toward enhancing institutional reputation. The publish or perish adage is age-old and has been given new life and impetus by institutional pressure on investigators who are not only concerned with their own academic advancement and reputation, but also increasingly required to earn substantial portions of their own salaries through continuously generating grants that support their academically based remuneration. Another facet of this phenomenon is in the number of co-authors per publication, which has grown substantially during recent decades. The growing complexity of multidisciplinary teamwork makes monitoring and adherence to scientific norms even more difficult. ${ }^{13}$

Building a culture of research quality and integrity requires discussion and standard-setting across small and large enterprises of varying complexity. Responsibility for ensuring the integrity of scientific processes and dissemination of reliable results falls on research professionals, funders, institutional administrators, scientific societies, journal editors, and clinicians. These entities have their own interests that can be in conflict, and their priorities and expectations can be at odds. Today's science is exciting and innovative, but its very complexity contributes to the risk of misconduct. ${ }^{14}$

\section{WHAT ARE THE SOLUTIONS FOR SURGEONS?}

Preventing misconduct and ensuring research integrity requires a set of research standards. A recent step in that direction was taken in this journal and 2 other major cardiothoracic surgery journals by the simultaneous publication of a proposed checklist for research integrity investigations, which also provides the necessary components of appropriate research conduct. ${ }^{4}$ The checklist, if used appropriately, is designed to address whether an investigation follows a reasonable process and if the institutional report 
of its investigation is appropriate and complete. Perhaps even more important than a checklist for use in investigations of misconduct allegations is the use of a checklist for the actual conduct of clinical research, as presented by Bando and colleagues ${ }^{15}$ along with the accompanying commentaries. This checklist is applicable to most types of clinical research, and we suggest that, with minor modifications, it could be applied to basic science and outcomes research. The components of the checklist are reasonable: standardized training for all investigators, fully reviewed scientific protocols with documentation of agreement by the investigators, data collection and storage standards, and numerous other suggestions for standardizing research processes. Adherence to these checklist components have the potential to help getting the research right in the first place and if not, ensuring that missteps will not happen again. ${ }^{15}$

Surgeons can undertake a number of other strategies to contribute to scientific integrity. First and foremost, research practices and policies should be updated by departments and institutions to align values, ideals, and expectations concerning research processes. Secondly, our institutions should foster a culture of research reliability and surgeons should educate ourselves and our trainees in the responsible conduct of research as well as in research ethics. ${ }^{16}$ Further to this, institutions should develop and employ a methodology that provides value to research that is not published but still advances the field or discipline. Thirdly, allegations of research misconduct that are brought to the attention of our departments, institutions, or journal editorial staffs must be taken seriously. Whistleblowers should be treated fairly in a timely manner, and be protected and thanked for coming forward with their concerns. Fourthly, institutions should establish a research integrity advisory board that includes key stakeholders, such as investigators and patient-subjects potentially influenced by the findings. Such advisory boards could be adopted by our journals, ensuring a fair, unbiased approach to policies relevant to allegations of research misconduct. Our surgical societies should have clearly expressed expectations about the integrity of research presented at their meetings, as well as disciplinary standards, including the consequences of transgressions. ${ }^{17}$ Responsibility for publishing an ethically challenged or flawed article ultimately rests on the editors, who must therefore be informed and educated about detecting, investigating, and responding to research misconduct. ${ }^{6,14,18}$ There is no substitute for surgeons to accept personal responsibility to learn and acquire the skills necessary for critically reviewing and appraising the quality of research and its integrity in all settings-within our own groups, departments, institutions, or journals. The required education is available and we should make it a priority to acquire it. ${ }^{18}$
Additionally, a special consideration is the way in which we interact with industry. Surgical investigators have access to industry funding for research studies that they later publish. We and our respective institutions must use industrial funding responsibly, and we must perform and publish the research with the highest level of integrity, regardless of funding sources, accepting no outside undue influence.

Whilst the recommendations we have described might seem demanding, momentum is growing nationally and internationally to produce truly hardwired and standardized accountability around research integrity. The European Promoting Integrity as an Integral Dimension of Excellence in Research project has conducted comprehensive studies of research processes, and a consensus panel produced clear outcomes and recommendations, which cover all aspects of research integrity: policy, education, training, cultural acceptance, incentive management, transparency, and safety, and establishment of a research integrity committee. ${ }^{16}$ There is support within the United States for the development of a national advisory board for research integrity that would support, enforce, and adjudicate in a manner similar to the recommendations of the Promoting Integrity as an Integral Dimension of Excellence in Research project. ${ }^{6}$ Each of us as scientists and surgeons can consider these recommendations and work toward adopting them in our institutions. ${ }^{19-21}$ Although each of us could develop our own research integrity program, collectively we can do even more by acting to improve the overall culture of maintaining research integrity. ${ }^{21}$

\section{CONCLUSIONS}

Integrity and trust are essential attributes of scientific and biomedical research. Scientific misconduct represents a danger to institutions as well as to their faculty, residents, students, staff, and patients, and even more importantly, to the entire body of science itself. To achieve and maintain public trust, scientists and surgeons must take steps to ensure and maintain the integrity of our research enterprise. To do so requires collaboration among researchers and among academic institutions. Our failure to maintain integrity in our research will lead to increasing efforts to do so by outside stakeholders, including funding organizations and the public and patients we serve. This could ultimately lead to undesirable consequences such as substantial financial and nonfinancial penalties, adverse publicity, and reputational damage. To ensure scientific integrity, researchers and institutions would be best served to self-educate, self-regulate, and ultimately self-reportthis is, after all, the central responsibility as well as the very definition of a profession. 


\section{Conflict of Interest Statement}

Authors have nothing to disclose with regard to commercial support.

\section{References}

1. Wakefield AJ, Murch SH, Anthony A, Linnell J, Casson DM, Malik M, et al. Ileal-lymphoid-nodular hyperplasia, non-specific colitis, and pervasive developmental disorder in children. Lancet. 1998;351:637-41.

2. Gerber JS, Offit PA. Vaccines and autism: a tale of shifting hypotheses. Clin Infect Dis. 2009;48:456-61.

3. Gunsalus CK, Marcus AR, Oransky I. Institutional research misconduct reports need more credibility. JAMA. 2018;319:1315-6.

4. Sade RM, Rylski B, Swain JA, Entwistle JWC, Ceppa DP, for the Cardiothoracic Ethics Forum. Institutional investigations of ethically flawed reports in cardiothoracic surgery journals. J Thorac Cardiovasc Surg. 2020;57:617-9.

5. Redman BK. Correctable myths about research misconduct in the biomedical sciences. Sci Eng Ethics. 2019;25:621-9.

6. Sade RM. Sanctions for research misconduct in cardiothoracic surgery journals. $J$ Thorac Cardiovasc Surg. 2016;152:661-3.

7. Robishaw JD, DeMets DL, Wood SK, Boiselle PM, Hennekens CH. Establishing and maintaining research integrity at academic institutions: challenges and opportunities. Am J Med. 2020;133:e87-90.

8. Grey A, Bolland M, Gamble G, Avenell A. Quality of reports of investigations of research integrity by academic institutions. Res Integr Peer Rev. 2019;4:3.

9. National Academies of Sciences, Engineering, and Medicine. Optimizing the Nation's Investment in Academic Research: A New Regulatory Framework for the $21^{\text {st }}$ Century. Washington, DC: National Academies Press; 2016.
10. National Academies of Sciences, Engineering, and Medicine. Fostering Integrity in Research. Washington, DC: National Academies Press; 2017.

11. Institute of Medicine. Evolution of Translational Omics: Lessons Learned and the Path Forward. Washington, DC: National Academies Press, 2012:122.

12. Davies SR. An ethics of the system: talking to scientists about research integrity. Sci Eng Ethics. 2019;25:1235-53.

13. Hussinger K, Pellens M. Scientific misconduct and accountability in teams. PLoS One. 2019;14:e0215962.

14. Gunsalus CK, McNutt MK, Martinson BC, Faulkner LR, Nerem RM. Overdue: a US advisory board for research integrity. Nature. 2019;566:173-5.

15. Bando K, Schaff HV, Sato T, Hashimoto K, Cameron DE. A multidisciplinary approach to ensure scientific integrity in clinical research. Ann Thorac Surg. 2015;100:1534-6.

16. Forsberg EM, Anthun FO, Bailey S, Birchley G, Bout H, Casonato C, et al. Working with research integrity-guidance for research performing organisations: the Bonn PRINTEGER statement. Sci Eng Ethics. 2018;24:1023-34.

17. Sade RM. Publication of unethical research studies: the importance of informed consent. Ann Thorac Surg. 2003;75:325-8.

18. Berling E, McLeskey C, O'Rourke M, Pennock RT. A new method for a virtuebased responsible conduct of research curriculum: pilot test results. Sci Eng Ethics. 2019;25:899-910.

19. Davidoff F. Checklists and guidelines: imaging techniques for visualization what to do. JAMA. 2010;304:206-7.

20. Tang FC, Steen RG, Casadevall A. Misconduct accounts for the majority of retracted scientific publications. Proc Nat Acad Sci U S A. 2012;109: 17028-33.

21. Iribarne A, McCullough JN. Addressing scientific integrity through education in the responsible conduct of research. Ann Thorac Surg. 2015;100:1538-9. 